\title{
THE SURVIVING RATE OF A GRAPH FOR THE FIREFIGHTER PROBLEM*
}

\author{
CAI LEIZHEN ${ }^{\dagger}$ AND WANG WEIFAN ${ }^{\ddagger}$
}

\begin{abstract}
We consider the following firefighter problem on a graph $G=(V, E)$. Initially, a fire breaks out at a vertex $v$ of $G$. In each subsequent time unit, a firefighter protects one vertex, and then the fire spreads to all unprotected neighbors of the vertices on fire. The objective of the firefighter is to save as many vertices as possible. Let $\operatorname{sn}(v)$ denote the maximum number of vertices the firefighter can save when a fire breaks out at vertex $v$ of $G$. We define the surviving rate $\rho(G)$ of $G$ to be the average percentage of vertices that can be saved when a fire randomly breaks out at a vertex of $G$, i.e., $\rho(G)=\sum_{v \in V} \operatorname{sn}(v) / n^{2}$. In this paper, we prove that for every tree $T$ on $n$ vertices, $\rho(T)>1-\sqrt{2 / n}$. Furthermore, we show that $\rho(G)>1 / 6$ for every outerplanar graph $G$, and $\rho(H)>3 / 10$ for every Halin graph $H$ with at least 5 vertices.
\end{abstract}

Key words. firefighter problem, surviving rate, tree, outerplanar graph, Halin graph

AMS subject classifications. 05C35, 05C90

DOI. $10.1137 / 070700395$

1. Introduction. The firefighter problem was introduced by Hartnell [9] in 1995 in an attempt to model the spread of fire, diseases, computer viruses, and similar phenomena at a macro-control level. A fire breaks out at a vertex of a graph $G=$ $(V, E)$ at time 0 . For each time interval $(i, i+1], i \geq 0$, a firefighter protects one vertex not yet on fire (the vertex remains protected afterwards), and the fire spreads from burning vertices (i.e., vertices on fire) to all unprotected neighbors of these vertices. The process ends when the fire can no longer spread, and all vertices that are not burning are saved. The objective of the firefighter is to save as many vertices as possible.

It is not surprising that the firefighter problem is very difficult. Indeed, it has been shown by Finbow et al. [5] that the problem is NP-complete even for trees of maximum degree 3. On the other hand, Hartnell and $\mathrm{Li}[10]$ have proved that a simple greedy method for trees is a 1/2-approximation algorithm, and MacGillivray and Wang [8] have given a $0-1$ integer programming formulation of the problem for trees and solved the problem in polynomial time for some subclass of trees. Recently, Cai, Verbin, and Yang [2] have obtained a $(1-1 / e)$-approximation algorithm for trees based on a linear programming relaxation and randomized rounding, and they have also considered fixed parameter tractability of the problem on trees. Furthermore, several authors (Develin and Hartke [3], Fogarty [7], and Wang and Moeller [11]) have considered various aspects of the problem for $d$-dimensional grids, especially the number of firefighters required to contain the fire. We refer the reader to a recent survey of Finbow and MacGillivray [6] for more information on the firefighter problem.

* Received by the editors August 17, 2007; accepted for publication (in revised form) July 1, 2009; published electronically December 9, 2009.

http://www.siam.org/journals/sidma/23-4/70039.html

${ }^{\dagger}$ Department of Computer Science and Engineering, The Chinese University of Hong Kong, Shatin, Hong Kong SAR, China (lcai@cse.cuhk.edu.hk). This author was partially supported by grant CUHK4165/01E from the Research Grants Council of Hong Kong.

${ }^{\ddagger}$ Institute of Mathematics, Zhejiang Normal University, Jinhua, Zhejiang 321004, China (wwf@ zjnu.cn). This author was partially supported by grant NSFC 10771197 of China. 
In this paper, we study the fire defending ability of a graph as a whole by considering the average percentage of vertices the firefighter can save. Let $\operatorname{sn}(v)$ denote the maximum number of vertices in $G$ that the firefighter can save when a fire breaks out at vertex $v$, which will be referred to as the surviving number for $v$. We define the surviving rate $\rho(G)$ of $G$ to be the average percentage of vertices that can be saved when a fire randomly breaks out at a vertex of the graph, i.e.,

$$
\rho(G)=\frac{\sum_{v \in V} \operatorname{sn}(v)}{n^{2}} .
$$

For example, the surviving rates of paths and cycles are $\rho\left(P_{n}\right)=1-\frac{2}{n}+\frac{2}{n^{2}}$ for $n \geq 2$ and $\rho\left(C_{n}\right)=1-\frac{2}{n}$. We note that the concept of surviving rates is closely related to the notion of expected damage introduced by Finbow et al. [4], who investigated graphs of minimum expected damage. To be precise, $\rho(G)=1-\operatorname{ed}(G) / n$, where $\operatorname{ed}(G)=\frac{1}{n} \sum_{v \in V}(n-\operatorname{sn}(v))$ is the expected damage $\operatorname{ed}(G)$ of $G$.

Our main results are the following lower bounds on surviving rates of trees, outerplanar graphs, ${ }^{1}$ and Halin graphs ${ }^{2}$ :

$$
\rho(G)> \begin{cases}1-\sqrt{2 / n} & \text { if } G \text { is a tree with } n \text { vertices, } \\ 1 / 6 & \text { if } G \text { is an outerplanar graph, } \\ 3 / 10 & \text { if } G \text { is a Halin graph with at least } 5 \text { vertices. }\end{cases}
$$

We will proceed as follows. We fix notation and give definitions in section 2 . We then establish lower bounds on the surviving rates of trees (section 3), outerplanar graphs (section 4), and Halin graphs (section 5). We will also discuss future directions and propose some open problems in section 6 .

2. Notation and definitions. All graphs in this paper are connected undirected simple graphs with $m$ edges and $n \geq 2$ vertices. For a graph $G=(V, E)$ and a vertex $v \in V, d(v)$ denotes the degree of $v$ in $G$, and $N(v)$ the set of neighbors of $v$, i.e., vertices adjacent to $v$. A $d$-vertex is a vertex of degree $d$, and we use $V_{d}$ and $n_{d}$, respectively, to denote the set and the number of $d$-vertices in $G$. We use $\Delta$ to denote the maximum degree of $G$.

For a subgraph $H$ of $G$, we use $\operatorname{sn}(H)=\sum_{v \in V(H)} \operatorname{sn}(v)$ to denote the surviving number for $H$, and $\overline{\operatorname{sn}}(H)=\operatorname{sn}(H) /|V(H)|$ to denote the average surviving number for $H$. We will also use the above notation and terms for a subset $V^{\prime}$ of vertices; e.g., $\operatorname{sn}\left(V^{\prime}\right)$ denotes the surviving number for $V^{\prime}$.

A rooted tree is a tree $T$ with one vertex $r$ chosen as the root. For each vertex $v$ in $T$, its neighbor on the unique $(r, v)$-path in $T$ is the parent of $v$, and each of the other neighbors of $v$ is a child of $v$. A vertex $u$ is a descendant of $v$ if $v$ is on the unique $(r, u)$-path in $T$. A rooted subtree $T_{v}$ of $T$ is the subtree of $T$ consisting of $v$, which is the root of $T_{v}$, and all other descendants of $v$. The height of a rooted tree $T_{r}$ is the length of a longest root-to-leaf path, and the height of a vertex $v$ in $T_{r}$ is the height of the rooted subtree $T_{v}$.

3. Trees. Let us start with the following simple observation: if the firefighter protects vertices $u_{1}, u_{2}, \ldots, u_{t}$ successively to save $k$ vertices in a graph $G$, then for

\footnotetext{
${ }^{1} \mathrm{~A}$ graph is an outerplanar graph if it has a planar embedding with all vertices on the boundary of the outer face.

${ }^{2} \mathrm{~A}$ graph is a Halin graph if it is formed from a planar embedding of a tree without vertices of degree 2 by connecting all its leaves by a cycle that crosses no edges.
} 
any spanning subgraph $H$ of $G$, he can protect these vertices in the same order to save at least $k$ vertices in $H$. This implies the following connection between surviving rates of a graph and its spanning subgraphs.

FACT 3.1. For any spanning subgraph $H$ of a graph $G$, we have $\rho(G) \leq \rho(H)$.

The above fact indicates that, among all graphs, trees have the highest surviving rates, as every connected graph contains a spanning tree. Since we can save at most $n-d(v)$ vertices when a fire breaks out at a vertex $v$, every tree $T$ satisfies

$$
\rho(T) \leq \frac{1}{n^{2}} \sum_{v \in V}(n-d(v))=1-\frac{2 m}{n^{2}}=1-\frac{2}{n}+\frac{2}{n^{2}} .
$$

We note that trees attaining this upper bound are exactly caterpillars ${ }^{3}$ where the distance between every pair of vertices of degree at least 3 is not equal to 2 , which was shown by Finbow et al. [4] in their study of graphs with minimum expected damage. Table 3.1 gives surviving rates of small trees.

TABLE 3.1

Surviving rates of trees with at most 9 vertices.

\begin{tabular}{|c|c|c|c|c|c|c|c|c|}
\hline$n$ & 2 & 3 & 4 & 5 & 6 & 7 & 8 & 9 \\
\hline$\rho(T)$ & $\frac{1}{2}$ & $\frac{5}{9}$ & $\frac{5}{8}$ & $\frac{17}{25}$ & $\frac{13}{18}$ & $\frac{36}{49}, \frac{37}{49}$ & $\frac{49}{64}, \frac{50}{64}$ & $\frac{63}{81}, \frac{64}{81}, \frac{65}{81}$ \\
\hline
\end{tabular}

In this section, we show that the surviving rate of every tree on $n$ vertices is greater than $1-\sqrt{2 / n}$, which tends to 1 as $n \rightarrow \infty$. To establish this lower bound, we first give two lemmas about surviving numbers for paths and subtrees in a tree.

Lemma 3.2. For every path $P$ on $k \geq 2$ vertices in a tree $T, \operatorname{sn}(P)>(k-2) n$.

Proof. Let $P=v_{1} v_{2} \cdots v_{k}$. Let $T_{i}$ denote the connected component of $T-$ $\left\{v_{i-1}, v_{i+1}\right\}$ containing vertex $v_{i}$, and $t_{i}$ the number of vertices in $T_{i}$. When a fire breaks out at vertex $v_{i}, 1 \leq i \leq k$, we protect $v_{i-1}$ first and then $v_{i+2}$ (do nothing if $v_{i-1}$ or $v_{i+2}$ does not exist) to save all vertices in $T-T_{i}-T_{i+1}$. Therefore $\operatorname{sn}\left(v_{i}\right) \geq$ $n-t_{i}-t_{i+1}$ and

$$
\begin{aligned}
\operatorname{sn}(P) & \geq \sum_{i=1}^{k}\left(n-t_{i}-t_{i+1}\right) \\
& =k n-\sum_{i=1}^{k}\left(t_{i}+t_{i+1}\right) \\
& >(k-2) n . \quad
\end{aligned}
$$

Lemma 3.3. For every rooted subtree $S$ of height $h \geq 0$ in a rooted tree $T$, $\operatorname{sn}(S) \geq(n-h-1)|S|$, where $|S|$ is the number of vertices in $S$.

Proof. When a fire breaks out at a vertex $v$ of $S$, we use the simple strategy of protecting its parent in $T$, which will save all vertices in $T-T_{v}$ (note that $T_{v}=S_{v}$ ). If $S=T$ and the fire breaks out at the root, we protect a child of the root.

\footnotetext{
${ }^{3} \mathrm{~A}$ caterpillar is a tree that has a path such that every vertex not in the path is adjacent to some vertex in the path.
} 
Let $H_{i}, 0 \leq i \leq h$, be the set of vertices of height $i$ in $S$. Then for each $0 \leq i \leq h$, $\sum_{v \in H_{i}} n_{v} \leq|S|$, where $n_{v}$ denotes the number of vertices in $T_{v}$. Therefore

$$
\operatorname{sn}\left(H_{i}\right)=\sum_{v \in H_{i}}\left(n-n_{v}\right) \geq\left|H_{i}\right| n-|S|,
$$

and thus

$$
\begin{aligned}
\operatorname{sn}(S) & =\sum_{i=0}^{h} \operatorname{sn}\left(H_{i}\right) \\
& \geq \sum_{i=0}^{h}\left(\left|H_{i}\right| n-|S|\right) \\
& =n \sum_{i=0}^{h}\left|H_{i}\right|-\sum_{i=0}^{h}|S| \\
& =(n-h-1)|S| .
\end{aligned}
$$

The above lemma implies that the surviving rate of a tree of small diameter is close to 1 , where the diameter $\operatorname{diam}(G)$ of a graph $G$ equals the maximum pairwise distance in $G$.

Corollary 3.4. For every $n$-vertex tree $T, \rho(T) \geq 1-\frac{\lceil\operatorname{diam}(T) / 2\rceil+1}{n}$.

Proof. Let $P=v_{0}, v_{1}, \ldots, v_{t}$ be a longest path in $T$ and $h=\lceil t / 2\rceil=\lceil\operatorname{diam}(T) / 2\rceil$. We can make $T$ a rooted tree of height $h$ by choosing $v_{h}$ as the root, and the bound follows directly from Lemma 3.3.

Now we establish the $1-\sqrt{2 / n}$ lower bound on the surviving rate of a tree. For a rooted tree on $n$ vertices, a long path is a path that contains at least $\sqrt{2 n}$ vertices, and a short tree is a rooted subtree whose height is at most $\sqrt{2 n}-2$. Clearly, if a rooted subtree does not contain a long path from the root to a leaf, then it is a short tree. The main idea in our proof is to partition a rooted tree into long paths and short trees, and then use Lemmas 3.2 and 3.3 to obtain a lower bound on the surviving rate of the tree. We choose $\sqrt{2 n}$ to define long paths in order to balance the lower bounds from these two lemmas.

TheOREM 3.5. For every $n$-vertex tree $T, \rho(T)>1-\sqrt{2 / n}$.

Proof. It suffices to prove the theorem for $n \geq 3$. We first make $T$ a rooted tree by arbitrarily choosing a vertex as its root, and then use induction on the height of $T$ to show that $T$ can be partitioned into long paths and short trees.

Let $P$ be a longest root-to-leaf path in $T$. If $P$ has less than $\sqrt{2 n}$ vertices, then the height of $T$ is at most $\sqrt{2 n}-2$. Therefore $T$ itself is a short tree, and we are done. Otherwise, we delete all vertices of $P$ from $T$ to obtain a forest $F$. Note that $F$ is a collection of rooted trees, and the choice of $P$ guarantees that every rooted subtree of any rooted tree in $F$ is also a rooted subtree of $T$. Furthermore, the height of any rooted tree in $F$ is one less than that of $T$. By the induction hypothesis, each rooted tree in $F$ can be partitioned into long paths and short trees. These partitions and $P$ together give us a required partition of $T$.

Let $\mathcal{P}$ be the set of long paths and $\mathcal{S}$ the set of short trees in the partition. Denote by $|P|$ the number of vertices in a path $P$, by $|S|$ the number of vertices in a short tree $S$, and by $h(S)$ the height of $S$. If $T$ itself is a short tree, the theorem follows directly from Lemma 3.3. Otherwise $|\mathcal{P}| \geq 1$, and it follows from Lemmas 3.2 and 3.3 
that

$$
\begin{aligned}
\operatorname{sn}(T) & =\sum_{P \in \mathcal{P}} \operatorname{sn}(P)+\sum_{S \in \mathcal{S}} \operatorname{sn}(S) \\
& >\sum_{P \in \mathcal{P}}(|P|-2) n+\sum_{S \in \mathcal{S}}(n-h(S)-1)|S| \\
& =\left(\sum_{P \in \mathcal{P}}|P|+\sum_{S \in \mathcal{S}}|S|\right) n-\left(\sum_{P \in \mathcal{P}} 2 n+\sum_{S \in \mathcal{S}}(h(S)+1)|S|\right) \\
& \geq n^{2}-\left(\sum_{P \in \mathcal{P}} 2 n+\sum_{S \in \mathcal{S}} \sqrt{2 n}|S|\right)
\end{aligned}
$$

as $\sum_{P \in \mathcal{P}}|P|+\sum_{S \in \mathcal{S}}|S|=n$ and $h(S) \leq \sqrt{2 n}-2$.

Let $p$ denote $\sum_{P \in \mathcal{P}}|P|$. Then $|\mathcal{P}| \leq\lfloor p /\lceil\sqrt{2 n}\rceil\rfloor \leq p / \sqrt{2 n}$, and it follows that

$$
\operatorname{sn}(T)>n^{2}-\left(\frac{p}{\sqrt{2 n}} \times 2 n+\sqrt{2 n}(n-p)\right)=n^{2}-\sqrt{2 n} n,
$$

which yields $\rho(T)>1-\sqrt{2 / n}$.

4. Outerplanar graphs. In this section we prove that the surviving rate of every outerplanar graph with at least 2 vertices is greater than $1 / 6$. For this purpose, we first give a lower bound on the surviving number for a vertex in a maximal outerplanar graph. ${ }^{4}$

Lemma 4.1. For every vertex $v$ of a maximal outerplanar graph $G$ with $n \geq 4$ vertices, we have $\operatorname{sn}(v) \geq \frac{n}{2(d(v)-1)}$.

Proof. Let $G^{*}$ be an outerplanar embedding of $G$. Then the boundary of the outer face of $G^{*}$ forms an $n$-cycle $C$. For two vertices $x$ and $y$ in $C$, let $C[x, y]$ denote the section of $C$ from $x$ to $y$, and let $C[x, y)=C[x, y] \backslash\{y\}$. Let $v$ be a $d$-vertex and $x_{1}, x_{2}, \ldots, x_{d}$ its neighbors ordered according to their orders in $C$.

Since $\left\{C\left[x_{i}, x_{i+1}\right): 1 \leq i<d\right\}$ partitions $C-\left\{v, x_{d}\right\}$ into $d-1$ sections, one section, say $C\left[x_{t}, x_{t+1}\right)$, contains at least $(n-2) /(d-1)$ vertices, and thus $C\left[x_{t}, x_{t+1}\right]$ contains at least $(n-2) /(d-1)+1$ vertices. If $\left|C\left[x_{t}, x_{t+1}\right]\right|=2$, then $d=n-1$ and the lemma is clearly true. Otherwise $\left|C\left[x_{t}, x_{t+1}\right]\right| \geq 3$, and by the maximality of $G$, there is a unique vertex $y_{t} \in C\left(x_{t}, x_{t+1}\right)$ that forms a 3 -face with vertices $x_{t}, x_{t+1}$ (see Figure 4.1).

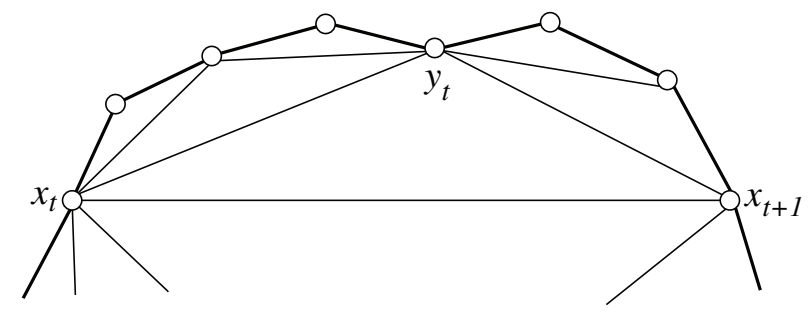

FIG. 4.1. Inside section $C\left[x_{t}, x_{t+1}\right]$.

\footnotetext{
${ }^{4} \mathrm{An}$ outerplanar graph is maximal if no edge can be added without losing outerplanarity.
} 
Clearly, one of $C\left[x_{t}, y_{t}\right]$ and $C\left[y_{t}, x_{t+1}\right]$, say $C\left[x_{t}, y_{t}\right]$, contains at least $(n-2) / 2(d-$ $1)+1$ vertices. When a fire breaks out at $v$, we protect $x_{t}$ first and then $y_{t}$ to save all vertices in $C\left[x_{t}, y_{t}\right]$. Therefore

$$
\operatorname{sn}(v) \geq \frac{n-2}{2(d-1)}+1=\frac{n+2 d-4}{2(d-1)} \geq \frac{n}{2(d-1)}
$$

as $d \geq 2$.

We now use the above lemma to establish the following lower bound on surviving rates of outerplanar graphs.

THEOREM 4.2. The surviving rate of every outerplanar graph with $\geq 2$ vertices is greater than $1 / 6$.

Proof. In light of Fact 3.1, it suffices to prove the theorem for every maximal outerplanar graph $G$ since every outerplanar graph is a spanning subgraph of some maximal outerplanar graph. Also we assume $n \geq 4$, as the theorem is clearly true for $n=2,3$.

Let $V_{d}$ be the set of $d$-vertices, and $n_{d}$ the number of $d$-vertices. By Lemma 4.1, we have

$$
\operatorname{sn}(G)=\sum_{d=2}^{\Delta} \operatorname{sn}\left(V_{d}\right) \geq \sum_{d=2}^{\Delta} \frac{n}{2(d-1)} n_{d}=\frac{1}{6} n \beta
$$

for $\beta=\sum_{d=2}^{\Delta} \frac{3}{d-1} n_{d}$.

To calculate the value of $\beta$, we note that $G$ contains $2 n-3$ edges, which implies $\sum_{v \in V} d(v)=4 n-6$. Therefore

$$
2 n_{2}+3 n_{3}+\cdots+\Delta n_{\Delta}=4\left(n_{2}+n_{3}+\cdots+n_{\Delta}\right)-6
$$

which yields

$$
\sum_{d=5}^{\Delta}(d-4) n_{d}+6=2 n_{2}+n_{3} .
$$

We now use (4.1) to show that $\beta>n$ :

$$
\begin{aligned}
\beta & =\sum_{d=2}^{\Delta} \frac{3}{d-1} n_{d} \\
& =3 n_{2}+\frac{3}{2} n_{3}+n_{4}+\sum_{d=5}^{\Delta} \frac{3}{d-1} n_{d} \\
& \geq n_{2}+n_{3}+n_{4}+\frac{1}{2}\left(2 n_{2}+n_{3}\right)+\sum_{d=5}^{\Delta} \frac{3}{d-1} n_{d} \\
& =n_{2}+n_{3}+n_{4}+\frac{1}{2}\left(\sum_{d=5}^{\Delta}(d-4) n_{d}+6\right)+\sum_{d=5}^{\Delta} \frac{3}{d-1} n_{d} \\
& =n_{2}+n_{3}+n_{4}+3+\sum_{d=5}^{\Delta}\left(\frac{d-4}{2}+\frac{3}{d-1}\right) n_{d} .
\end{aligned}
$$

Since $\frac{d-4}{2}+\frac{3}{d-1}>1$ for $d \geq 5$, we have $\beta>n+3$ and therefore

$$
\rho(G)=\operatorname{sn}(G) / n^{2}>\frac{1}{6} .
$$


5. Halin graphs. In this section we show that the surviving rate of every Halin graph with $n \geq 5$ vertices is greater than $3 / 10$. Recall that a Halin graph is formed from a planar embedding of a tree $T$ without 2 -vertices by connecting all its leaves by a cycle $C$ that crosses no edges. We note that a Halin graph contains at least 4 vertices and at most $2 n-2$ edges. Table 5.1 gives surviving rates of small Halin graphs.

TABLE 5.1

Surviving rates of Halin graphs with at most 8 vertices.

\begin{tabular}{|c|c|c|c|c|c|}
\hline$n$ & 4 & 5 & 6 & 7 & 8 \\
\hline$\rho(G)$ & $\frac{1}{4}$ & $\frac{9}{25}$ & $\frac{11}{36}, \frac{12}{36}$ & $\frac{19}{49}, \frac{20}{49}$ & $\frac{28}{64}, \frac{29}{64}, \frac{30}{64}$ \\
\hline
\end{tabular}

We assume that a planar embedding of a Halin graph $G$ is given where all vertices in the cycle $C$ lie on the outer face. All vertices not on $C$, i.e., nonleaf vertices of the tree $T$, are internal vertices, and neighbors of a vertex are ordered in a counterclockwise direction. We also use the following legend for the figures in this section: the source of a fire is indicated by a black vertex, a vertex on fire is shaded, a protected vertex is enclosed by a square box, and the time a vertex is on fire or protected is given by a number beside the vertex.

To obtain a lower bound on the surviving rate of a Halin graph, we start with two lemmas on the surviving number for a vertex. The first lemma shows that some vertices on the cycle $C$ cause very little damage to the graph when they are sources of fire, and the second lemma gives a lower bound on the surviving number for a vertex in terms of its degree.

Lemma 5.1. The cycle $C$ of every Halin graph $G$ contains at least two vertices such that the surviving number for each vertex is at least $n-5$.

Proof. The lemma is trivially true for $n \leq 6$, and thus we may assume $n \geq 7$. Let $T^{\prime}$ be the tree obtained from the tree $T$ of $G$ by removing all leaves of $T$, i.e., vertices of the cycle $C$ of $G$.

Case 1. $T^{\prime}$ has a leaf $v$ with $d_{G}(v) \geq 4$. If $d_{G}(v)=4$, then, as illustrated in Figure 5.1(a), the leftmost and rightmost neighbors of $v$ on $C$ both have surviving numbers at least $n-4$. Otherwise, $d_{G}(v) \geq 5$ and, as illustrated in Figure 5.1(b), the second leftmost and second rightmost neighbors of $v$ on $C$ both have surviving numbers at least $n-4$.

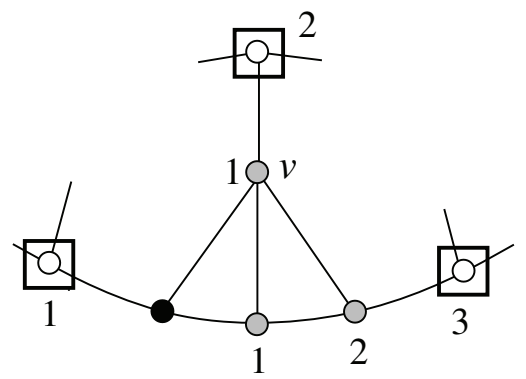

(a)

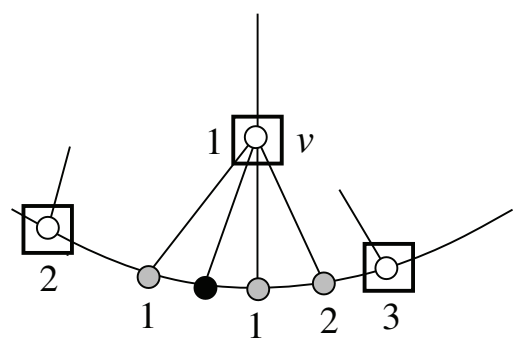

(b)

FIG. 5.1. (a) The situation for $d_{G}(v)=4$ up to symmetry, and (b) the situation for $d_{G}(v) \geq 5$ up to symmetry (the rightmost vertex may or may not be a neighbor of $v$ ). 
Case 2. All leaves of $T^{\prime}$ have degree 3 in $G$. Let $P$ be a longest path in $T^{\prime}$. Since $G$ has at least 7 vertices, $T^{\prime}$ contains at least 2 vertices. We claim that for each end of $P$ there is a distinct vertex on $C$ whose surviving number is at least $n-5$. Let $v$ be an arbitrary end of $P$, and $u$ the unique vertex in $T^{\prime}$ that is adjacent to $v$. Order the neighbors $N_{G}(u)$ of $u$ in $G$ counterclockwise. Let $x \in N_{G}(u)$ be the vertex immediately preceding $v$, and $y \in N_{G}(u)$ the vertex immediately following $v$. If one of $x, y$, say $x$, is on $C$, then we have the situation shown in Figure 5.2(a), and the surviving number for vertex $z$ in the figure is at least $n-4$. Otherwise, both $x$ and $y$ are vertices of $T^{\prime}$. Since $v$ is an end of a longest path in $T^{\prime}$, one of $x, y$, say $x$, is a leaf of $T^{\prime}$, and we have the situation shown in Figure 5.2(b). As illustrated in the figure, the surviving number for vertex $z$ is at least $n-5$.

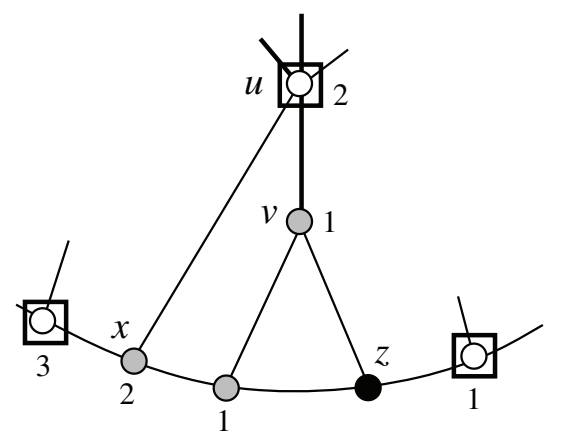

(a)

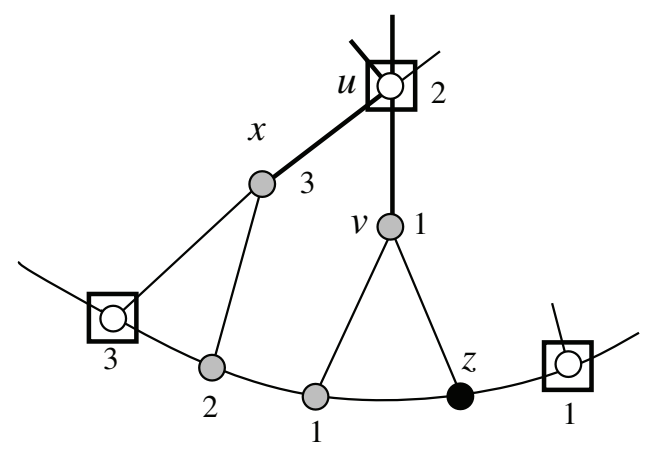

(b)

FIG. 5.2. (a) Vertex $x$ is on the cycle $C$, and (b) vertex $x$ is a leaf of $T^{\prime}$. Edges of $T^{\prime}$ are indicated by thick lines.

Lemma 5.2. Let $G$ be a Halin graph with $n \geq 7$ vertices. Then for every vertex $v$ in $G, \operatorname{sn}(v) \geq \frac{n-1}{d(v)}$.

Proof. We consider two cases, depending on whether vertex $v$ is in the cycle $C$.

Case 1. $v$ is a vertex in $C$. Let $u$ be the unique neighbor of $v$ in $T$, and regard $T-v$ as a tree rooted at $u$. Order the subtrees rooted at the children of $u$ counterclockwise. Call the first one the leftmost subtree, and the last one the rightmost subtree. Note that $d(v)=3$ and we need to show that $\operatorname{sn}(v) \geq(n-1) / 3$.

Case 1.1. One of the leftmost and rightmost subtrees of $u$ contains at least $(n-1) / 3$ vertices.

Without loss of generality, we may assume that the rightmost subtree $T^{\prime}$ of $u$ contains at least $(n-1) / 3$ vertices. If the cycle $C$ has at least two more vertices from $v$ to the leftmost vertex of $T^{\prime}$, we can save all vertices in $T^{\prime}$ by protecting its rightmost vertex, root, and leftmost vertex in this order (see Figure 5.3(a)), which implies $\operatorname{sn}(v) \geq(n-1) / 3$. Otherwise, $C$ has only one vertex from $v$ to the leftmost vertex of $T^{\prime}$, and thus $T^{\prime}$ contains $n-3$ vertices. We first protect the rightmost vertex of $T^{\prime}$ and then the root of $T^{\prime}$. At this point, the leftmost vertex of $T^{\prime}$ is on fire. From now on, we contain the fire to spread along the cycle $C$ counterclockwise by protecting the unique internal neighbor $w$ of the vertex $x$ most recently on fire until we have the situation that $w$ has been protected already. When this happens, we protect the right neighbor $x^{\prime}$ of $x$ on $C$ (do nothing if $x^{\prime}$ has been protected already), and the fire can no longer spread (see Figure 5.3(b)). Therefore we save more than half of the vertices in $T^{\prime}$, and thus $\operatorname{sn}(v)>(n-3) / 2 \geq(n-1) / 3$ for $n \geq 7$. 


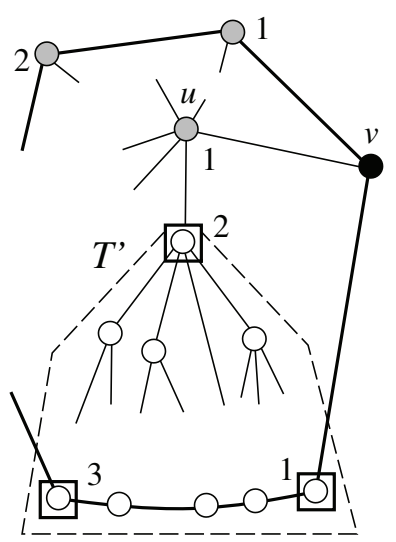

(a)

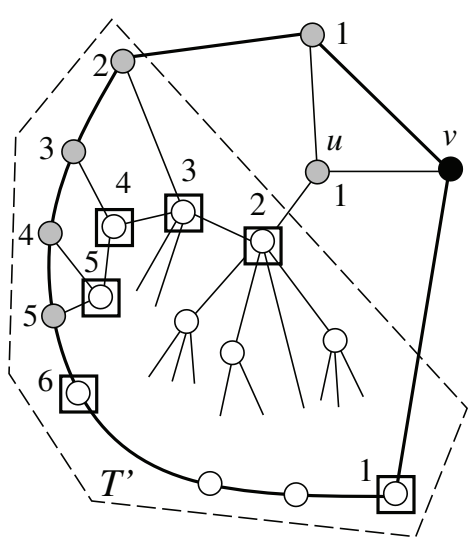

(b)

Fig. 5.3. The situation for the case that the rightmost subtree $T^{\prime}$ contains at least $(n-1) / 3$ vertices: (a) $C$ contains at least two vertices from $v$ to the leftmost vertex of $T^{\prime}$, and (b) $C$ contains only one vertex from $v$ to the leftmost vertex of $T^{\prime}$.

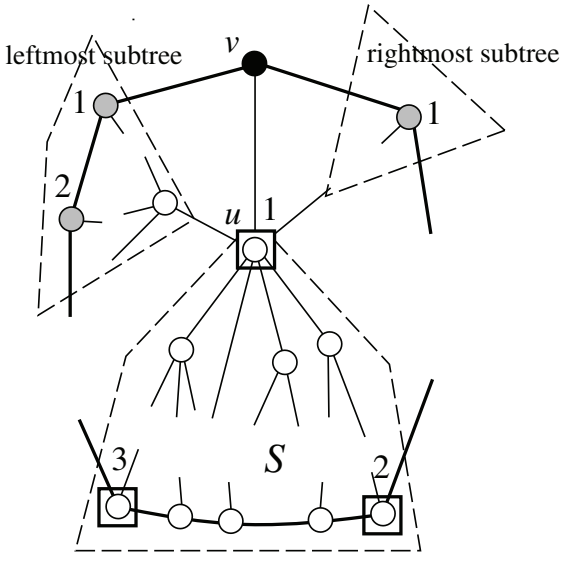

(a)

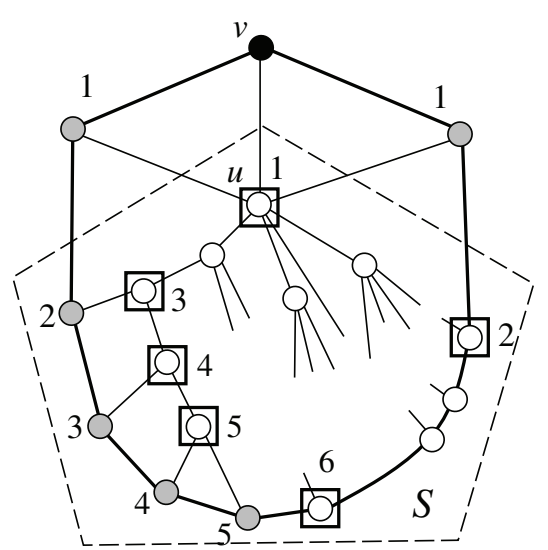

(b)

FIG. 5.4. The situation for the case that both the leftmost and rightmost subtrees contain fewer than $(n-1) / 3$ vertices: (a) one of the leftmost and rightmost subtrees contains more than one vertex, and (b) both the leftmost and rightmost subtrees contain one vertex.

Case 1.2. Both the leftmost and rightmost subtrees of $u$ contain fewer than $(n-1) / 3$ vertices. In this case, let $S$ denote the tree rooted at $u$ after deleting both the leftmost and rightmost subtrees of $u$ from $T-v$. Then $S$ contains at least $(n-1) / 3 \geq 2$ vertices as $n \geq 7$. Without loss of generality, we may assume that the leftmost subtree of $u$ contains at least as many vertices as the rightmost subtree of $u$. If the leftmost subtree of $u$ contains at least two vertices, then $C$ contains at least two more vertices from $v$ to the leftmost vertex of $S$, and we can save all vertices in $S$ by protecting vertices in the order shown in Figure 5.4(a), implying $\operatorname{sn}(v) \geq(n-1) / 3$. Otherwise, both the leftmost and rightmost subtrees of $u$ contain one vertex, and thus $S$ has $n-3$ vertices. We protect vertex $u$ first, then the rightmost vertex in $S$. At 


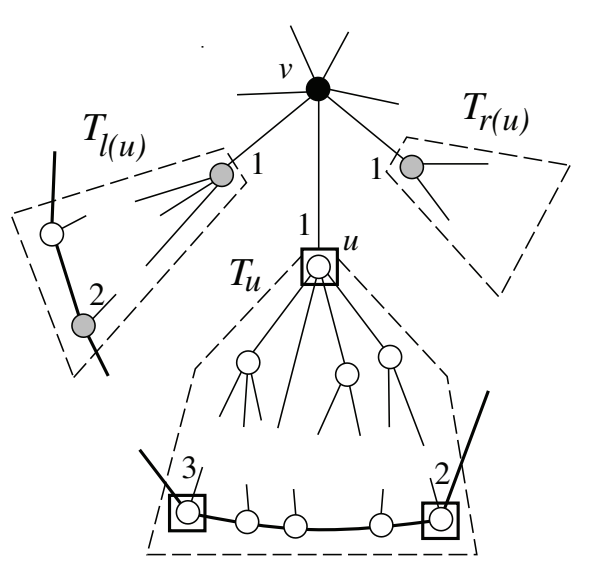

(a)

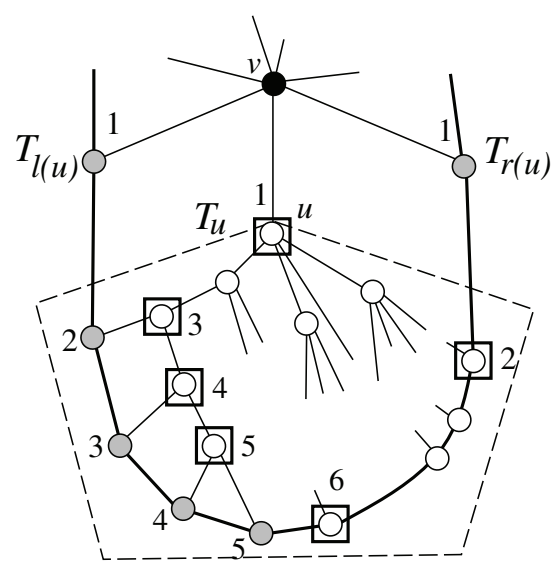

(b)

Fig. 5.5. Case 2. $v$ is an internal vertex: (a) one of the leftmost and rightmost subtrees contains more than one vertex, and (b) both the leftmost and rightmost subtrees contain one vertex.

this point, the leftmost vertex of $S$ is on fire, and we protect vertices in $S$ as we did for $T^{\prime}$ in Case 1.1 to contain the fire to spread along the cycle $C$ counterclockwise (see Figure 5.4(b)). Therefore we save more than half of the vertices in $S$, and thus $\operatorname{sn}(v)>(n-3) / 2 \geq(n-1) / 3$ for $n \geq 7$.

Case 2. $v$ is not a vertex in $C$.

In this case, $v$ is an internal vertex of $T$. Regard $T$ as a tree rooted at $v$, and order subtrees rooted at the children of $v$ counterclockwise around $v$. By pairing up each subtree with the subtree on its right, we get $d(v)$ pairs of subtrees with a total number of $2(n-1)$ vertices. Therefore there is a pair $T_{u}, T_{r(u)}$ of subtrees with at least $2(n-1) / d(v)$ vertices. Without loss of generality, we may assume that $T_{u}$ contains at least as many vertices as $T_{r(u)}$. Then $T_{u}$ contains at least $(n-1) / d(v)$ vertices. Let $T_{l(u)}$ denote the subtree to the left of $T_{u}$. If one of $T_{l(u)}$ and $T_{r(u)}$, say $T_{l(u)}$, contains at least two vertices, we can save all vertices in $T_{u}$ by first protecting $u$, then the rightmost vertex of $T_{u}$, followed by the leftmost vertex of $T_{u}$ (see Figure 5.5(a)). Otherwise, both $T_{l(u)}$ and $T_{r(u)}$ contain one vertex, and thus $T_{u}$ contains at least $2(n-1) / d(v)-1$ vertices since $T_{u} \cup T_{r(u)}$ has at least $2(n-1) / d(v)$ vertices. We first protect vertex $u$ and then the rightmost vertex of $T_{u}$. At this point, the leftmost vertex in $T_{u}$ is on fire, and we deal with $T_{u}$ as what we did for $T^{\prime}$ in Case 1.1 to contain the fire to spread along the cycle $C$, counterclockwise (see Figure 5.5(b)). Therefore we save at least $1+\left(n_{u}-1\right) / 2$ vertices in $T_{u}$, where $n_{u}$ is the number of vertices in $T_{u}$, which yields $\operatorname{sn}(v) \geq(n-1) / d(v)$.

We now use Lemmas 5.1 and 5.2 to obtain a lower bound on the surviving rate of a Halin graph.

THEOREM 5.3. For every Halin graph $G$ with $n \geq 5$ vertices, its surviving rate $\rho(G)>\frac{3}{10}$.

Proof. From Table 5.1, we see that the theorem holds for $5 \leq n \leq 8$, and thus we assume $n \geq 9$. We also note that the number of leaves in an $n$-vertex tree equals

$$
\sum_{d=3}^{\Delta}(d-2) n_{d}+2
$$


where $n_{d}$ is the number of $d$-vertices in the tree. This is easily derived from the following relation:

$$
\sum_{d=1}^{\Delta} d n_{d}=2(n-1)=2\left(\sum_{d=1}^{\Delta} n_{d}-1\right) .
$$

We are now ready to establish the lower bound in the theorem. By Lemma 5.1, the cycle $C$ contains two vertices $x, y$ such that each of them has surviving number at least $n-5$. Let $V^{\prime}=V(C)-\{x, y\}$. Since the vertices in the cycle $C$ of $G$ are exactly the leaves of the tree $T$ of $G$, we have

$$
\left|V^{\prime}\right|=|V(C)|-2=\sum_{d=3}^{\Delta}(d-2) n_{d} .
$$

Therefore each internal vertex $v$ can be matched with $d(v)-2$ distinct vertices $V^{\prime}(v)$ in $V^{\prime}$, and we consider the surviving number for $\{v\} \cup V^{\prime}(v)$ collectively.

Note that vertices in $V^{\prime}$ are 3 -vertices, and every internal vertex has degree at least 3. By Lemma 5.2, we have $\operatorname{sn}(v) \geq(n-1) / d(v)$ for each vertex $v$. Therefore the average surviving number

$$
\begin{aligned}
\overline{\operatorname{sn}}\left(\{v\} \cup V^{\prime}(v)\right) & \geq \frac{1}{d(v)-1}\left(\frac{n-1}{d(v)}+\frac{n-1}{3} \times(d(v)-2)\right) \\
& =\frac{n-1}{3}\left(1+\frac{2}{d(v)-1}-\frac{3}{d(v)}\right) .
\end{aligned}
$$

In the interval $[3, \infty)$, function $f(x)=1+2 /(x-1)-3 / x$ monotonically decreases in $[3,3+\sqrt{6})$ and monotonically increases in $(3+\sqrt{6}, \infty)$. Since $3+\sqrt{6} \approx 5.45, f(d(v))$ reaches its minimum at $d(v)=5$ or 6 , which is $9 / 10$ for both 5 and 6 . It follows that

$$
\overline{\operatorname{sn}}\left(\{v\} \cup V^{\prime}(v)\right) \geq \frac{3}{10}(n-1) .
$$

Combining this with the fact that $\operatorname{sn}(x) \geq n-5$ and $\operatorname{sn}(y) \geq n-5$, we have

$$
\begin{aligned}
\operatorname{sn}(G) & \geq \frac{3}{10}(n-1)(n-2)+2(n-5) \\
& =\frac{3}{10} n^{2}+\left(\frac{11}{10} n-\frac{47}{5}\right) \\
& >\frac{3}{10} n^{2} \quad(\text { as } n \geq 9),
\end{aligned}
$$

and hence $\rho(G)>\frac{3}{10}$ for $n \geq 5$.

6. Concluding remarks. In this paper, we have introduced the notion of the surviving rate of a graph for the firefighter problem to measure the fire defending ability of a graph as a whole, and studied surviving rates of trees, outerplanar graphs, and Halin graphs.

Trees, outerplanar graphs, and Halin graphs all have constant lower bounds on their surviving rates. In other words, for every graph in these graph classes, we can save, on average, at least a constant percentage of vertices when a fire breaks out at a 
vertex. It is natural to ask whether other graph classes have this property as well. In particular, one may wish to generalize this property to planar graphs. Unfortunately, for planar graphs $K_{2, n}, \lim _{n \rightarrow \infty} \rho\left(K_{2, n}\right)=0$. Nevertheless, we feel that this property holds for planar graphs of maximum degree 3 .

CONJECTURE 6.1. There is a positive constant $c$ such that every nontrivial planar graph $G$ of maximum degree 3 satisfies $\rho(G) \geq c$.

On the other hand, we can also generalize the surviving rate of a graph to the $k$-surviving rate by allowing the firefighter to protect $k \geq 2$ vertices each time, which brings us the following problem for planar graphs.

Problem 6.2. Determine the minimum $k$ for which planar graphs have a constant lower bound on their $k$-surviving rates.

The lower bounds of surviving rates that we have obtained for trees, outerplanar graphs, and Halin graphs are not tight. It would be interesting to improve these lower bounds. However, this may require new techniques, instead of detailed analysis. For outerplanar graphs and Halin graphs, it is unclear whether their asymptotic surviving rates are 1 . We note that there are infinitely many outerplanar graphs ( $n$-fans, for instance) and Halin graphs ( $n$-wheels, for instance) $G$ with $\rho(G)$ approaching 1 as $n \rightarrow \infty$.

Problem 6.3. For outerplanar graphs (Halin graphs, respectively) $G$ on $n$ vertices, determine whether $\lim _{n \rightarrow \infty} \rho(G)=1$.

We have shown that the surviving rate of an $n$-vertex tree $T$ is at least $1-\sqrt{2 / n}$, which approaches 1 as $n$ tends to infinity. We conjecture that actually $\rho(T)$ approaches 1 much more quickly as follows.

Conjecture 6.4. For every n-vertex tree $T, \rho(T) \geq 1-\Theta\left(\frac{\log n}{n}\right)$.

In terms of algorithmic and complexity issues, we certainly expect that it is very hard to determine the surviving rate of a graph, but it seems also difficult to obtain an NP-completeness proof.

ConjeCture 6.5. It is NP-complete to determine the surviving rate of a tree.

We also note that the proofs of our theorems imply polynomial-time approximation algorithms for surviving rates of trees, outerplanar graphs, and Halin graphs. In particular, our discussions in section 3 imply a polynomial-time $(1-\sqrt{2 / n})$ approximation algorithm for the surviving rate of a tree. On the other hand, it follows directly from a result of Hartnell and $\mathrm{Li}[10]$ that the following greedy method for trees is a 1/2-approximation algorithm for the surviving rate of a tree: every time the firefighter protects a vertex that cuts off the maximum number of vertices from the fire. We feel that this greedy algorithm achieves a much better approximation ratio for surviving rates, and leave its performance analysis as the last open problem of the paper.

Problem 6.6. Determine the approximation ratio of the greedy algorithm for the surviving rate of a tree. Is it actually equal to $1-\Theta\left(\frac{\log n}{n}\right)$ ?

Note added in proof. Recently, Cai et al. [1] have proved Conjecture 6.4, and solved Problem 6.3 for outerplanar graphs and Problem 6.6.

\section{REFERENCES}

[1] L. Cai, Y. Cheng, E. Verbin, And Y. Zhou, Surviving rates of trees and outerplanar graphs for the firefighter problem, manuscript, 2009.

[2] L. CAI, E. VERBIN, AND L. YANG, Firefighting on trees: $(1-1 / e)$-approximation, fixed parameter tractability and a subexponential algorithm, in Proceedings of ISAAC 2008, S.-H. Hong, H. Hagamochi, and T. Fukunaga, eds., Lecture Notes in Comput. Sci. 5369, Springer, New York, 2008, pp. 258-269. 
[3] M. Develin and S. Hartke, Fire containment in grids of dimension three and higher, Disc. Appl. Math., 155 (2007), pp. 2257-2268.

[4] S. Finbow, B. Hartnell, Q. Li, And K. Schmeisser, On minimizing the effects of fire or a virus on a network, J. Combin. Math. Combin. Comput., 33 (2000), pp. 311-322.

[5] S. Finbow, A. King, G. MacGillivray, and R. Rizzi, The firefighter problem for graphs of maximum degree three, Disc. Math., 307 (2007), pp. 2094-2105.

[6] S. Finbow and G. MacGillivray, The firefighter problem: A survey of results, directions, and questions, Australas. J. Combin., 43 (2009), pp. 57-78.

[7] P. Fogarty, Catching the Fire on Grids, M.Sc. Thesis, Department of Mathematics, University of Vermont, Burlington, VT, 2003.

[8] G. MacGillivray and P. Wang, On the firefighter problem, J. Combin. Math. Combin. Comput., 47 (2003), pp. 83-96.

[9] B. Hartnell, Firefighter! An application of domination, presentation at the 25th Manitoba Conference on Combinatorial Mathematics and Computing, University of Manitoba, Winnipeg, Canada, 1995.

[10] B. Hartnell AND Q. LI, Firefighting on trees: How bad is the greedy algorithm?, in Proceedings of the Thirty-first Southeastern International Conference on Combinatorics, Graph Theory and Computing (Boca Raton, FL, 2000), Congr. Numer. 145, Utilitas Math., Winnipeg, MB, 2000, pp. 187-192.

[11] P. Wang and S. Moeller, Fire control on graphs, J. Combin. Math. Combin. Comput., 41 (2002), pp. 19-34. 